\title{
Influence of a dielectric layer on photon emission induced by a scanning tunneling microscope
}

\author{
X. Tao, ${ }^{1}$ Z. C. Dong, ${ }^{1, a)}$ J. L. Yang, ${ }^{1}$ Y. Luo, ${ }^{1}$ J. G. Hou, ${ }^{1, a)}$ and J. Aizpurua ${ }^{2}$ \\ ${ }^{1}$ Hefei National Laboratory for Physical Sciences at the Microscale, University of Science and Technology \\ of China, Hefei, Anhui 230026, China \\ ${ }^{2}$ Centro Mixto de Física de Materiales CSIC-UPV/EHU and Donostia International Physics Center (DIPC), \\ Paseo Manuel de Lardizabal 4, Donostia-San Sebastián 20018, Spain
}

(Received 30 October 2008; accepted 8 January 2009; published online 27 February 2009)

\begin{abstract}
We investigate theoretically the influence of a dielectric layer on light emission induced by a scanning tunneling microscope through a combined approach of classical electrodynamics and first-principles calculations. The modification of the junction geometry upon the insertion of a dielectric layer is treated first by using the density functional theory to calculate the effective potential along the surface normal and then by solving a one-dimensional Schrödinger equation to obtain the exact distance between the tip and the substrate for a given current and bias voltage. The modified external field with the inclusion of a dielectric layer is evaluated by using the Fresnel formula. The local-field enhancement factor and radiated power are calculated by the boundary element method for two typical systems, W-tip/ $\mathrm{C}_{60} / \mathrm{Au}(111)$ and $\mathrm{W}$-tip $/ \mathrm{Al}_{2} \mathrm{O}_{3} / \mathrm{NiAl}(110)$. The calculated results indicate that the insertion of a dielectric layer tends to reduce the light emission intensity considerably but hardly changes the spectral profile with no substantial peak shifts with respect to the layer-free situation, in agreement with experimental observations. The suppression of the radiated power is mainly due to the increase in the tip-metal separation and the resultant reduction in the electromagnetic coupling between the tip and metal substrate. (C) 2009 American Institute of Physics. [DOI: 10.1063/1.3080766]
\end{abstract}

\section{INTRODUCTION}

A scanning tunneling microscope (STM) is capable of more than just observing and manipulating the nanoworld with atomic resolution; the tunneling current can also be used as a local source of excitation to produce light from the junction, which can provide additional information on local electromagnetic properties pertaining to the decay of various excitations. Photon emission from metal surfaces excited by tunneling electrons is attributed to the radiative decay of localized, collective plasmon modes between the tip and the sample. ${ }^{1}$ The intensity and spectrum of the emitted light depend mainly on two factors: The current fluctuations associated with inelastic electron tunneling and the strength of coupling between tunneling electrons and the electromagnetic field in the vicinity of the tip. The high frequency component of tunneling currents can drive charge density oscillations confined between the tip and the sample and act as a source for the radiation. ${ }^{2,3}$ Such interface plasmons are generally called tip-induced plasmons (TIPs), ${ }^{1}$ with a lateral extension of several nanometers. ${ }^{4}$ The TIP resonance leads to strong electromagnetic coupling between the tip and the sample. ${ }^{1,5}$ The electric field in the proximity of tip is greatly enhanced due to both the localization at the tip apex and the strong interaction of the surface charges of opposite polarity on the two electrodes. ${ }^{3,6}$ The strength of this local-field enhance-

\footnotetext{
${ }^{a)}$ Authors to whom correspondence should be addressed. Electronic addresses: zcdong@ustc.edu.cn and jghou@ustc.edu.cn.
}

ment is determined by the junction geometry and the dielectric functions of both tip and sample materials.

While the theory for photon emission from metal surfaces have been well developed using either a nanosphere model $^{2,3}$ or a hyperbolic tip geometry, ${ }^{7,8}$ the influence of the presence of a dielectric layer on light emission in STM has not been explored in so much detail, amounting to mainly the work by Amemiya ${ }^{9}$ who employed the vector spherical wave expansion method to consider the effect of a dielectric layer on STM induced luminescence. Nevertheless, the enhancement effect predicted in Ref. 9 is in contradiction with weakened emission observed in most experiments, ${ }^{10,11}$ probably due to the assumption of a fixed tip-substrate distance $(1 \mathrm{~nm})$ regardless of the existence of a dielectric layer. The light emission intensity is known to depend critically on the junction geometry, particularly the gap distance. But the exact calculation of the gap distance for a given current is not trivial when a dielectric layer is inserted, and no literature report has been found so far on this issue. In addition, the role of a dielectric layer on plasmon mediated emission from metal surfaces (suppression or enhancement) remains to be theoretically addressed.

Another reason for the present study stems from the ever-increasing interest in metal-enhanced fluorescence, ${ }^{12-14}$ single molecular electroluminescence, ${ }^{15,16}$ and plasmonenhanced spectroscopy. ${ }^{17-19}$ A dielectric spacer layer such as thin oxide,${ }^{11}$ halide ${ }^{20}$ and molecular layers ${ }^{21}$ has been used to suppress fluorescence quenching via charge transfer and nonradiative energy transfer so that molecular based fluorescence can be generated near metal surfaces by STM. There- 
fore, theoretical understanding of how a thin dielectric layer will modify plasmon mediated emissions is also important for the development of plasmonics.

In the present work, we propose a combined approach of first-principles calculations with classical electrodynamics to elucidate the effect of a dielectric layer on STM induced light emission. While first-principles calculations based on a density functional theory (DFT) is used to describe the potential of the vacuum-layer-metal system when a dielectric layer is inserted, classical electrodynamics is used to describe the electromagnetic fields at the STM cavity. Our calculated results can reproduce nicely the experimental observations. The agreement is achieved through the exact calculation of the gap distance between the tip and the metal substrate for a given set of tunnel current and bias voltage when a dielectric layer is inserted, taking into account the modification of the junction potential profile and voltage drop.

We use DFT to calculate the effective potential along the surface normal for a preset gap distance and then solve the Schrödinger equation to obtain the wave functions and their corresponding tunnel currents. Moreover, the effect of the dielectric layer on the local electric field is also considered. We use the Fresnel formula to calculate the reflection coefficients at each interface and add all beams of the coherent light together with a phase difference to evaluate the total external field. In addition, unlike the spherical tip geometry and the vector spherical wave expansion method used in Ref. 9 , a more realistic hyperbolic tip shape and the boundary element $\operatorname{method}^{8}$ (BEM) are used to calculate the local electric field enhancement at the STM cavity in the nonretarded limit. The optical properties of light emission are calculated using the reciprocity theorem of electrodynamics.

We investigated two typical systems, one with a molecular dielectric layer $\left[\mathrm{W}\right.$-tip/ $\left.\mathrm{C}_{60} / \mathrm{Au}(111)\right]$ and the other with an oxide dielectric layer [W-tip $\left./ \mathrm{Al}_{2} \mathrm{O}_{3} / \mathrm{NiAl}(110)\right]$. We found that the radiated power is reduced in comparison with the emission from the pristine metal surface but the spectral profile remains very similar without substantial peak shifts. We demonstrate in this paper that the correct description of the electronic density of the dielectric layer is a key element to properly describe the potential profile across the tunnel junction and the resultant dependence of the current on the separation distance at the cavity, and finally to obtain the right light emission rates.

\section{MODEL AND METHODS}

\section{A. Calculation of gap distance for a given current}

The calculation of intensity of emitted light can be separated into two parts: The power spectrum of current fluctuations $\left|I_{\omega}\right|^{2}$ and the local-field enhancement factor $G$. The former $\left(\left|I_{\omega}\right|^{2}\right)$ can be calculated using a free-electron model to describe the tip and metal substrate, ${ }^{22}$ while the latter $(G)$ is closely related to the junction geometry and is very sensitive to the tip-sample distance. Therefore, the calculation of the exact distance between the tip and the sample for a given set of tunnel current and bias voltage is very important.

The inclusion of a dielectric layer (hereafter termed as a

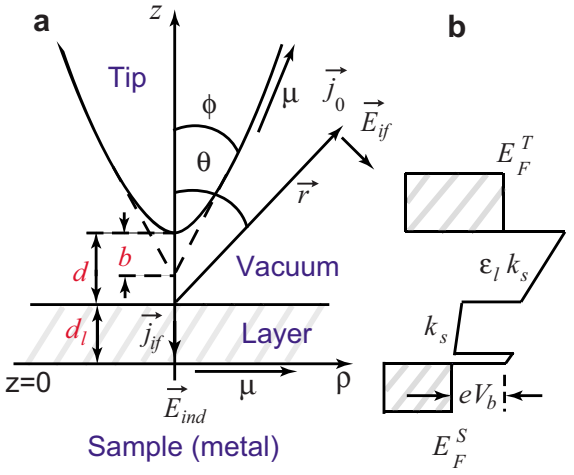

FIG. 1. (Color online) (a) The geometry for the STM configuration with a dielectric layer. The curvature of the apex is given by the ratio $b / d$, the aperture of the tip is given by the angle $\phi$ while the impinging angle $\theta$ is defined as the angle between $\vec{r}$ and $z$ axis. The bottom of the layer is chosen to be the $z=0$ plane. (b) The schematic energy of the system. $E_{F}^{T}$ and $E_{F}^{S}$ are the Fermi energies of the tip and the metal substrate, respectively. See texts for details.

layer for simplicity) into the tunnel junction is likely to modify (usually activate) certain tunneling channels and thus alter the gap distance between the tip and the metal substrate for a given current (Fig. 1). The modification introduced by the presence of the layer can be evaluated through the description of the potential profile across the junction. The first step is to calculate the potential distribution extending from the metal surface into the layer and vacuum. The DFT method via the plane wave code VASP (Ref. 23) is used to calculate the three-dimensional potential of the vacuumlayer-metal system. (The layer here can be either a dielectric layer or simply vacuum.) Averaging this potential over the $\hat{x}$ and $\hat{y}$ directions, we obtain the one-dimensional potential $V_{S}(z)$ along the surface normal. For a preset separation $(d$ $+d_{l}$ ) between the tip and the metal substrate where $d$ is the vacuum gap distance and $d_{l}$ is the thickness of the dielectric layer (Fig. 1), the potential must be matched across the junction and at the tip side $\left(z=d+d_{l}\right)$. We assume that the layer and the vacuum gap can each be treated as one-dimensional parallel-plate capacitors. ${ }^{24}$ Under such equivalent capacitance assumption, we can get the electrostatic distribution in the gap between the tip and the metal substrate. The overall potential from the metal substrate to the tip is defined as the effective potential $V_{\text {eff }}(z)$, which can be expressed as

$$
V_{\mathrm{eff}}(z)= \begin{cases}z k_{s}+V_{S}(z), & 0<z<d_{l}, \\ {\left[z+d_{l} / \varepsilon_{l}-d_{l}\right] \varepsilon_{l} k_{s}+V_{S}(z),} & d_{l}<z<d+d_{l},\end{cases}
$$

where $k_{s}=\left(e V_{b}+\Phi_{T}-\left.V_{S}(z)\right|_{z=d+d_{l}}\right) /\left(d_{l}+d \varepsilon_{l}\right)$ is the slope of the voltage drop across the layer and $\varepsilon_{l} k_{s}$ is the slope of the voltage drop across the vacuum gap; $\Phi_{T}$ is the workfunction of the tip, $V_{b}$ is the applied bias voltage, and $\varepsilon_{l}$ is the dielectric constant of the dielectric layer. The wave functions of electrons across the whole junction can be calculated using $V_{\text {eff }}(z)$. Let $V$ represent the quantization volume, and the wave functions will have the form ${ }^{3}$ 


$$
\psi=V^{-1 / 2} f(z) \exp \left[i \vec{k}_{\|} \cdot \vec{\rho}\right],
$$

where $f(z)$ is the one-dimensional wave function, $\vec{k}_{\|}$and $\vec{\rho}$ are the electron wave vector and position vector parallel to the surface, respectively. The function $f(z)$ can thus be determined by the one-dimensional Schrödinger equation with the potential $V_{\text {eff }}(z)$

$$
\left(-\frac{\hbar^{2}}{2 m_{e}} \frac{d^{2}}{d z^{2}}+V_{\mathrm{eff}}(z)\right) f(z)=E_{\perp} f(z),
$$

where $E_{\perp}$ is the energy perpendicular to the surface, $m_{e}$ is the electron mass, and $\hbar$ is the Planck constant. Equation (3) can be solved by the numerical method to obtain the wave functions and further to calculate the tunneling probability $T\left(E_{\perp}\right)$ of an electron from the tip to the metal substrate. Thus, the tunneling current $I$ can be expressed as follows: ${ }^{25,26}$

$$
I=\frac{2 e A_{\mathrm{eff}}}{(2 \pi)^{3}} \iint d^{2} k_{\|} \int d k_{\perp}\left(\frac{1}{\hbar} \frac{\partial E}{\partial k_{\perp}}\right) T\left(E_{\perp}\right),
$$

in which $(1 / \hbar)\left(\partial E / \partial k_{\perp}\right)$ is the group velocity of an electron perpendicular to the surface, and $A_{\text {eff }}$ is the effective tunneling area and is assumed to be $4 \mathrm{~nm}^{2}$ in the present calculations. Therefore, through the procedure described above, the tunneling current can be calculated for a given separation between the tip and the metal substrate, and vice versa. For a given tunnel current, we can calculate the exact gap distance using a recursive method.

\section{B. Radiated power}

In order to better evaluate the effect of both curvature and aperture of a tip on light emission, we use a hyperbolic tip geometry (Fig. 1) to describe the tip coordinate $[\rho(\mu), z(\mu)],{ }^{7}$ in which $\mu$ is a parameter describing the geometry going from 0 to $\pi / 2$. The radius of the tip curvature is $R=b \tan ^{2}(\phi)$, in which the distance $b$ is a parameter describing the sharpness of the very end of the tip, and the angle $\phi$ refers to the overall tip aperture (Fig. 1). ${ }^{7}$ The electrons tunneling from the tip to the sample give rise to the position- and energy-dependent current densities $\vec{j}_{i f}\left(\vec{r}^{\prime}, \omega\right)$, associated with the inelastic transition between an initial state at the tip and a final state at the sample (or vice versa, depending on the bias polarity). This current density is responsible for the radiated electric field $\vec{E}_{i f}(\vec{r}, \omega)$ at the observation point (detector at infinite). The direct calculation of the electric field $\vec{E}_{i f}(\vec{r}, \omega)$ is rather challenging; therefore, the reciprocity theorem is used to calculate the light emission spectra instead. A trial point current density $\vec{j}_{0} \delta^{(3)}(\vec{x}-\vec{r})$ is placed at the observation point and the induced electric field $\vec{E}_{\text {ind }}\left(\vec{r}, \vec{r}^{\prime}, \omega\right)$ is calculated below the tip at $r^{\prime}$. By employing the reciprocity theorem, the radiated electric field can be expressed as follows:

$$
\vec{E}_{i f}(\vec{r}, \omega)=\frac{i \omega}{4 \pi \varepsilon_{0} c^{2}} \frac{e^{i k r}}{r} \int d^{3} r^{\prime} G\left(\theta, \overrightarrow{r^{\prime}}, \omega\right) \vec{j}_{i f}\left(\vec{r}^{\prime}, \omega\right),
$$

where $c$ is the speed of light and $G\left(\theta, \overrightarrow{r^{\prime}}, \omega\right)$ represents the local-field enhancement at $\vec{r}^{\prime}$ for a plane wave impinging with angle $\theta$.
The total radiated power per unit solid angle $\Omega$ and per unit photon energy $\hbar \omega$ is

$$
\begin{aligned}
& \frac{d^{2} P}{d \Omega d(\hbar \omega)} \\
& =2 c \varepsilon_{0} \sum_{i, f} r^{2}\left|\vec{E}_{i f}(\vec{r}, \omega)\right|^{2} \delta\left(E_{i}-E_{f}-\hbar \omega\right), \\
& =\frac{\omega^{2}}{8 \pi^{2} \varepsilon_{0} c^{3}} \sum_{i, f}\left|\int d^{3} r^{\prime} G\left(\theta, \vec{r}^{\prime}, \omega\right) \vec{j}_{i f}\left(\vec{r}^{\prime}, \omega\right)\right|^{2} \delta\left(E_{i}-E_{f}\right. \\
& \quad-\hbar \omega) .
\end{aligned}
$$

Since TIPs are essentially localized below the tip, we can approximate Eq. (6) by taking the local enhancement factor $G$ out of the integral and obtain the following expression for the radiated power:

$$
\frac{d^{2} P}{d \Omega d(\hbar \omega)}=\frac{1}{2 \hbar} \frac{\omega^{2} d^{2}}{(4 \pi)^{2} \varepsilon_{0} c^{3}}\left|I_{\omega}\right|^{2}|G(\omega)|^{2} .
$$

The radiated power from the cavity of a STM in the presence of a layer can therefore be approximated by the convolution of the inelastic current intensity $I_{\omega}$ with the local-field enhancement $G$.

\section{Current density and local-field enhancement factor}

TIPs are driven by the inelastic scattering of the current $\left(\left|I_{\omega}\right|^{2}\right)$, which is the Fourier transform of the time-dependent part of the current correlation. ${ }^{2}$ At zero temperature within the usual transfer-Hamiltonian framework, the power spectrum of current using a free-electron model can be written $\mathrm{as}^{22}$

$$
\left|I_{\omega}\right|^{2}= \begin{cases}\frac{e I_{0}}{2 \pi}\left(1-\frac{\hbar \omega}{e V_{b}}\right), & 0 \leq \hbar \omega \leq e V_{b}, \\ 0, & \hbar \omega>e V_{b},\end{cases}
$$

where $I_{0}$ is the elastic tunnel current $(\omega=0)$. It is worthy to mention that the sophisticated calculation of the power spectrum using the wave functions determined by the detailed potential above may give results that are more precise in numbers but offer essentially the same physical picture. ${ }^{3}$ However, the numerical calculation of the real wave functions and their differentiations as well as the integration over space to get power spectra are extremely time consuming. Since the power spectrum of current is fixed at two extremes $\left(\hbar \omega=0\right.$ and $\left.\hbar \omega=e V_{b}\right)$ and no transitions between discrete energy levels of molecules are considered to contribute to the photon emission in the present calculation, the free-electron model used above should be enough to describe the power spectrum of the present system.

Upon substitution of Eq. (8) into Eq. (7), we can get the radiated power ${ }^{9}$

$$
\frac{d^{2} P}{d \Omega d(\hbar \omega)}=\frac{\omega^{2} e I_{0}}{(4 \pi)^{3} \hbar \varepsilon_{0} c^{3}} d^{2}\left(1-\frac{\hbar \omega}{e V_{b}}\right)|G(\omega)|^{2} .
$$

In order to calculate the local-field enhancement $G(\omega)$ upon insertion of a dielectric layer, the BEM is employed to cal- 


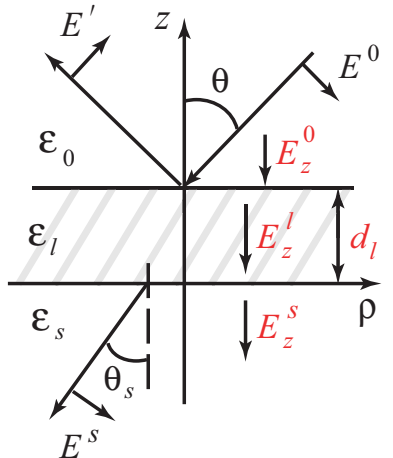

FIG. 2. (Color online) Schematics of the fields considered for the calculation of the external fields. $E_{z}^{0}, E_{z}^{l}$, and $E_{z}^{s}$ are the $\hat{z}$ components of the external field in the vacuum, the layer, and the metal substrate, respectively. $E^{0}$ and $E^{\prime}$ are the electric fields of the incidence and reflection plane waves, respectively, while $E^{S}$ is the electric field of the plane wave transmitting through the dielectric layer.

culate the charge distribution and the resultant field enhancement factor at the three interfaces across the junction with the presence of the hyperbolic tip described above (Fig. 1). ${ }^{7}$ By solving a self-consistent surface integral equation, we can obtain the surface charge density $\sigma(\vec{s}, \omega)$ induced at each interface separating two different mediums in the following way: ${ }^{7}$

$$
\Lambda \sigma(\vec{s}, \omega)=\vec{n}_{s} \cdot \nabla \varphi^{\operatorname{ext}}(\vec{s}, \omega)-\int \frac{\vec{n}_{s} \cdot\left(\vec{s}-\vec{s}^{\prime}\right)}{\left|\vec{s}-\vec{s}^{\prime}\right|^{3}} \sigma\left(\overrightarrow{s^{\prime}}, \omega\right) d \overrightarrow{s^{\prime}},
$$

where $\Lambda=2 \pi\left\{\left[\varepsilon_{2}(\omega)-\varepsilon_{1}(\omega)\right] /\left[\varepsilon_{2}(\omega)+\varepsilon_{1}(\omega)\right]\right\}$, the vector $\vec{s}$ and $\overrightarrow{s^{\prime}}$ refer to the interface points, $\vec{n}_{s}$ refers to the unit vector normal to the interface at point $\vec{s}$, and $\varphi^{\operatorname{ext}}(\vec{s}, \omega)$ is the external electrical potential acting at $\vec{s} . \varepsilon_{1}$ and $\varepsilon_{2}$ are the dielectric functions surrounding each interface point $\vec{s}$, to the left and right of the direction of the increasing parametrization $(\mu)$, respectively.

$-\nabla \varphi^{\operatorname{ext}}(\vec{s}, \omega)$ describes the $\omega$ component of the external field and has to be calculated first in order to solve Eq. (10). Figure 2 illustrates the model used to calculate the external field as a result of all the reflections and refractions in the double-layered structure when a dielectric film is inserted, as one choice of external fields that are closer to the real situation (only the tip is introduced after the external fields are calculated). It should be noted that other choices of the external fields considering metal surfaces alone or only incident fields could also work for the determination of the surface charge density as long as the local-field enhancement factor is scaled by the appropriate external field.

In the following, the parameters with superscript "0" refer to the vacuum, "l" refer to the layer, and $s$ refer to the metal substrate. Due to the characteristics of the driving current (perpendicular to the sample), only $p$-polarized component is considered here. Suppose a plane wave of $p$-polarization with electric field $\vec{E}^{0}$ illuminating on the system. By means of recurrence, the reflection coefficient of the interface between the vacuum and the layer can be obtained through Fresnel formula ${ }^{27}$

$$
r=\frac{E^{\prime}}{E^{0}}=\frac{r_{1}^{+}+r_{2}^{+} \exp \left[-2 i \delta_{l}\right]}{1+r_{1}^{+} r_{2}^{+} \exp \left[-2 i \delta_{l}\right]},
$$

where $r_{1}^{+}$and $r_{2}^{+}$are the Fresnel reflection coefficients for the vacuum-layer interface and the layer-substrate interface, respectively; $\delta_{l}=(2 \pi / \lambda) n_{l} d_{l} \cos \theta_{l}$ is usually called the phase thickness of a film, and $2 \delta_{l}$ is the phase difference between two adjacent beams of coherent light in the film. Thus, the $\hat{z}$ component of the external field in the vacuum can be expressed as

$$
\frac{E_{z}^{0}}{E^{0}}=(1-r) \sin \theta
$$

Accordingly, the transmission coefficient of the system is ${ }^{27}$

$$
t=\frac{E^{s}}{E^{0}}=\frac{t_{1}^{+} t_{2}^{+} \exp \left[-i \delta_{l}\right]}{1+r_{1}^{+} r_{2}^{+} \exp \left[-2 i \delta_{l}\right]},
$$

in which $t_{1}^{+}$and $t_{2}^{+}$are the transmission coefficients for the vacuum-layer interface and the layer-substrate interface, respectively, both with the layer being a semi-infinite material.

Similarly, the $\hat{z}$ component of the external field in the layer can be written as

$$
\frac{E_{z}^{l}}{E^{0}}=\frac{\left(1-r_{2}^{+}\right)(1+r) \tan \theta_{l} \cos \theta}{\left(1+r_{2}^{+}\right)}
$$

and for the metal substrate, the $\hat{z}$ component of the external field is given by

$$
\frac{E_{z}^{s}}{E^{0}}=t \tan \theta_{s}
$$

where

$$
\cos \theta_{l}=\sqrt{1-\frac{\varepsilon_{0}}{\varepsilon_{l}} \sin ^{2} \theta}, \quad \cos \theta_{s}=\sqrt{1-\frac{\varepsilon_{0}}{\varepsilon_{s}} \sin ^{2} \theta} .
$$

The radial components of the external field in the three regions are the same

$$
E_{\rho}^{0}=E_{\rho}^{l}=E_{\rho}^{s}=(1+r) \cos \theta .
$$

Thus, the external field at any position across the junction is obtained. By solving Eq. (10) with these calculated external fields for the three interfaces of our system (Fig. 1), we can obtain the surface charge density $\sigma(\vec{s}, \omega)$ induced at the surface of the cavity.

The proximity of a STM tip to the dielectric layer will generate an induced electric field at the cavity, $-\nabla \varphi^{\text {ind }}(\vec{r}, \omega)$, which can be calculated by

$$
-\nabla \varphi^{\operatorname{ind}}(\vec{r}, \omega)=\int \frac{\left(\vec{r}-\overrightarrow{s^{\prime}}\right)}{\left|\vec{r}-\overrightarrow{s^{\prime}}\right|^{3}} \sigma\left(\overrightarrow{s^{\prime}}, \omega\right) d \overrightarrow{s^{\prime}} .
$$

As a result, the local-field enhancement $G(\omega)$ produced can be obtained for each point $z$ along the cavity

$$
G_{z_{0}}(\omega)=\left.\frac{\hat{z} \cdot\left[\nabla \varphi^{\operatorname{ext}}(\vec{r}, \omega)+\nabla \varphi^{\mathrm{ind}}(\vec{r}, \omega)\right]}{\hat{z} \cdot\left[\nabla \varphi^{\operatorname{ext}}(\vec{r}, \omega)\right]}\right|_{z=z_{0}} .
$$

In Eq. (18), we have already scaled the local-field enhancement factor $G$ by the appropriate external field we got from 


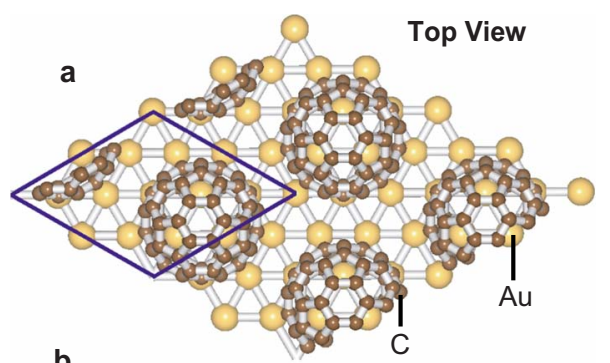

b

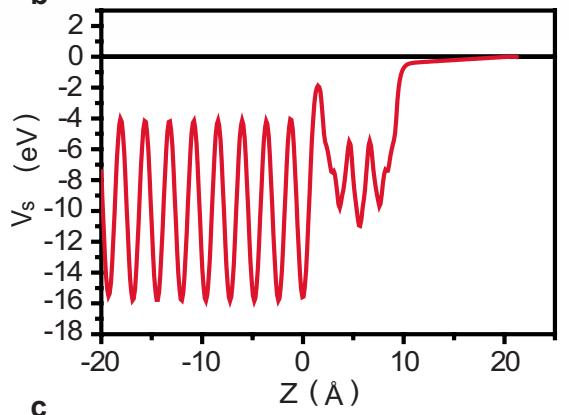

C

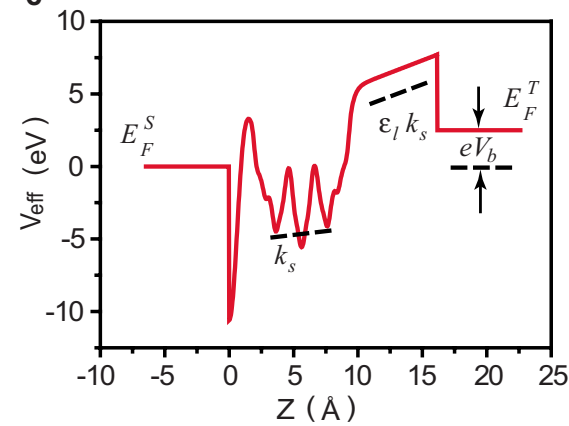

FIG. 3. (Color online) (a) The model used for the calculation of the onedimensional potential of the W-tip/ $\mathrm{C}_{60} / \mathrm{Au}(111)$ system. (b) The calculated result of $V_{S}(z)$. (c) The calculated result of the effective potential $V_{\text {eff }}(z)$.

the Fresnel formula [Eq. (12)] to avoid counting twice for the Fresnel coefficients. The radiated power can thus be calculated by substitution of Eq. (18) into Eq. (9).

\section{RESULTS AND DISCUSSION}

\section{A. W-tip/ $\mathrm{C}_{60} / \mathrm{Au}(111)$}

The first typical system we investigate is a tungsten tip on top of a molecular dielectric layer adsorbed on a metal surface, $\mathrm{W}$-tip $/ \mathrm{C}_{60} / \mathrm{Au}(111)$. The one-dimensional potential across the whole junction is calculated using the hexagonal $(2 \sqrt{3} \times 2 \sqrt{3}) R 30^{\circ}$ overlayer structure with $\mathrm{C}_{60}$ molecules adsorbed on the hcp sites of the $\mathrm{Au}(111)$ surface, ${ }^{28}$ as shown in Fig. 3(a). The metal substrate is modeled by a nine-layer slab. The density functional calculations for the wave functions were performed using the plane wave code VASP with a cutoff energy of $400 \mathrm{eV}$. The Perdew-Burke-Ernzerhof version $^{29}$ of the generalized gradient approximation is used for the treatment of electron exchange-correlation effects. Figures 3(b) and 3(c) show the calculated potentials of $V_{S}(z)$ and $V_{\text {eff }}(z)$ for $1 \mathrm{ML}$ of $\mathrm{C}_{60}$ adsorbed on $\mathrm{Au}(111)$, respectively. $V_{S}=0$ refers to the vacuum level, $V_{\text {eff }}=0$ refers to the Fermi level of the sample, and $z=0$ refers to the position of the top layer of the Au substrate. A bias voltage of $2.5 \mathrm{~V}$ with a tunnel current of $1.0 \mathrm{nA}$ was used in the calculation of the separation distance between the tip and the sample.
TABLE I. Calculated results of the separation distances between the tip and the sample of the W-tip $/ \mathrm{C}_{60} / \mathrm{Au}(111)$ system at $2.5 \mathrm{~V}$ and $1 \mathrm{nA}$. The last column lists the peak positions $\left(\lambda_{\max }\right)$ of the corresponding STM induced light emission spectra.

\begin{tabular}{ccccc}
\hline \hline The number of $\mathrm{C}_{60}$ layers & $\begin{array}{c}d_{l} \\
(\AA)\end{array}$ & $\begin{array}{c}d \\
(\AA)\end{array}$ & $\begin{array}{c}d_{l}+d \\
(\AA)\end{array}$ & $\begin{array}{c}\lambda_{\max } \\
(\mathrm{nm})\end{array}$ \\
\hline 0 & 0.000 & 7.502 & 7.502 & 593 \\
1 & 8.851 & 7.290 & 16.141 & 605 \\
2 & 16.361 & 6.894 & 23.255 & 608 \\
\hline \hline
\end{tabular}

As shown in Table I, the gap distance between the tip and the metal substrate $\left(d+d_{1}\right)$ is greatly increased on the insertion of $\mathrm{C}_{60}$ layers; however, the vacuum gap $(d)$ is reduced only slightly. The shortening of the vacuum gap distance is presumably due to the lower conductivity of thin $\mathrm{C}_{60}$ layers relative to $\mathrm{Au}$. It should be noted that the values reported here may underestimate the real conductivity difference because the density of states of $\mathrm{C}_{60}$ is not explicitly taken into account in the present calculation, which otherwise would make the vacuum gap distance smaller.

For the calculation of the local-field enhancement factor $G$, we choose $b=3.0 \mathrm{~nm}$ and $\phi=45^{\circ}$ to describe the tip geometry, so the radius of curvature of the tip is $R=b \tan ^{2}(\phi)$ $=3.0 \mathrm{~nm}$ (Fig. 1). We also choose the impinging angle $\theta$ $=45^{\circ}$ like in Refs. 3 and 7. The dielectric functions of Au and $\mathrm{W}$ are taken from Refs. 30 and 31, respectively. The dielectric constant of the $\mathrm{C}_{60}$ film is chosen to be $\varepsilon_{l}=4.4{ }^{32}$ Figure 4 shows the calculated spectra of light emission for 1 and 2 $\mathrm{ML}$ of $\mathrm{C}_{60}$ molecules adsorbed on Au. The peak position for the pristine $\mathrm{Au}$ substrate (layer-free) is around $593 \mathrm{~nm}$, which is consistent with both experiments and previous calculations $^{5,21}$ and thus justifies the validity of our method. (The wavelength dependent sensitivity of the detection system has been taken into account in the present and subsequent spectral plots.) A quantum efficiency of about 3.1 $\times 10^{-4}$ photons/electron is obtained for the $1 \mathrm{ML}$ of $\mathrm{C}_{60}$ on Au using a W-tip, which is about $59 \%$ of that for the pristine $\mathrm{Au}(111)$ substrate (for the integrated range of wavelength over $500-1100 \mathrm{~nm})$.

The primary feature of Fig. 4 is the pronounced decrease in emission intensity upon the insertion of a dielectric layer, in agreement with most experiments. ${ }^{10}$ The thicker the dielectric layer, the lower the emission intensity. The suppres-

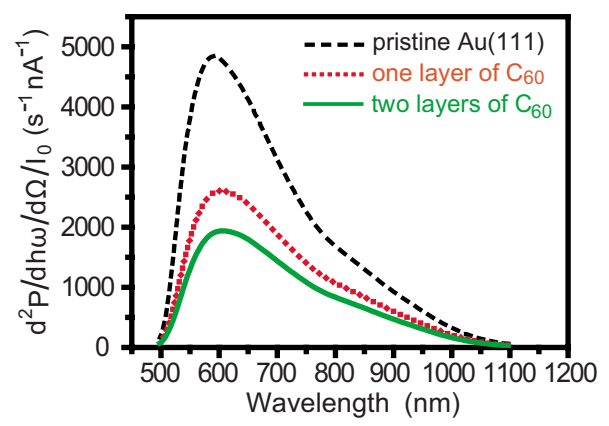

FIG. 4. (Color online) Light emission spectra for the pristine $\mathrm{Au}(111)$ (dashed) and the surfaces covered by 1 ML (dotted) and 2 ML (solid) of $\mathrm{C}_{60}$ molecules, respectively. The tunneling conditions are $2.5 \mathrm{~V}$ and $1 \mathrm{nA}$. 
sion of emission is attributed to the decrease in the local-field enhancement when a dielectric layer is inserted. In comparison with the layer-free situation, the distance between the tip and the metal surface is greatly increased on the insertion of the $\mathrm{C}_{60}$ layers. As a result, the electric field in the tip region, induced by the charge distribution at the metal-layer and vacuum-layer interfaces, is not as strong as that of the layerfree geometry even if the two situations have similar induced charge densities at tip and sample. Moreover, in practical situations, the induced charge density of the tip surface is also considerably reduced due to the increased separation and resultant weakened electromagnetic coupling between the tip and metal substrate when a dielectric layer is inserted, thus leading to further reduction in the local-field enhancement effect. It should be noted that enhanced plasmon mediated emission has also been reported in the $\mathrm{HBDC} / \mathrm{Cu}$ system, ${ }^{33}$ in which the molecules are somehow electronically decoupled from the metal substrate by bulky legs and so the dynamic dipole moments of molecules may add in to cause resonance enhancement through competing with the role of molecules as a geometrical spacer. ${ }^{34,35}$

Another feature in the emission spectra of Fig. 4 is the nearly the same spectral profile for both the pristine $\mathrm{Au}(111)$ and the surfaces covered with $\mathrm{C}_{60}$ molecules. Nevertheless, as shown in Table I, a small redshift is still discernable, 12 $\mathrm{nm}$ and $15 \mathrm{~nm}$ for one layer and two layers of $\mathrm{C}_{60}$ molecules relative to the layer-free situation, respectively. Such small peak shifts were also observed experimentally in HBDC molecules on $\mathrm{Cu}(111) \cdot{ }^{33}$ It is known that the spectral profile and peak position of emission spectra depend on both the geometry and the dielectric response of the junction. For the layer-free case, the relation between the lowest energy dipolar mode of TIP and the width of vacuum gap $d$ can be approximated as $\hbar \omega \sim d^{1 / n}$ ( $n$ being tip geometry dependent) when the tip radius is much larger than the gap distance $(R$ $\gg d$ ), which would lead to a blueshift for increased $d .{ }^{4}$ However, the effect of the gap distance becomes more complicated when a dielectric layer is inserted: First, the above approximation (in particular, the $R \gg d$ condition) may become questionable when the distance between the tip and metal surface is greatly increased. Second, the gap distance is no longer well-defined due to the induced charge density at both the metal-layer and layer-vacuum interfaces, resulting in a complicated combined effect of both the vacuum gap distance $(d)$ and the whole gap distance $\left(d+d_{l}\right)$. On the other hand, the inclusion of a dielectric layer into the junction will increase the value of the effective dielectric constants of the tip-metal substrate region, screening the polarization of the surface induced charges at the cavity, thus shifting the localized cavity plasmons to the red, as normal dielectric screening does. ${ }^{2,36}$ The observed shift is a combined result of the above effects with both tendencies (blue- and redshift) appearing to compensate each other.

\section{B. W-tip/ $\mathrm{Al}_{2} \mathrm{O}_{3} / \mathrm{NiAl}(110)$}

Oxide thin films are widely used as a decoupling layer in optoelectronic studies near surfaces. The second typical system we investigate is $\mathrm{W}$-tip $/ \mathrm{Al}_{2} \mathrm{O}_{3} / \mathrm{NiAl}(110)$ (Ref. 11) with
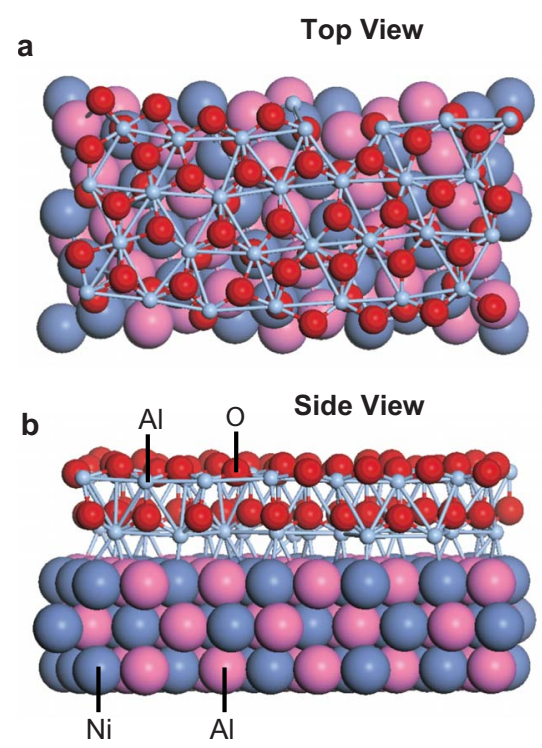

C
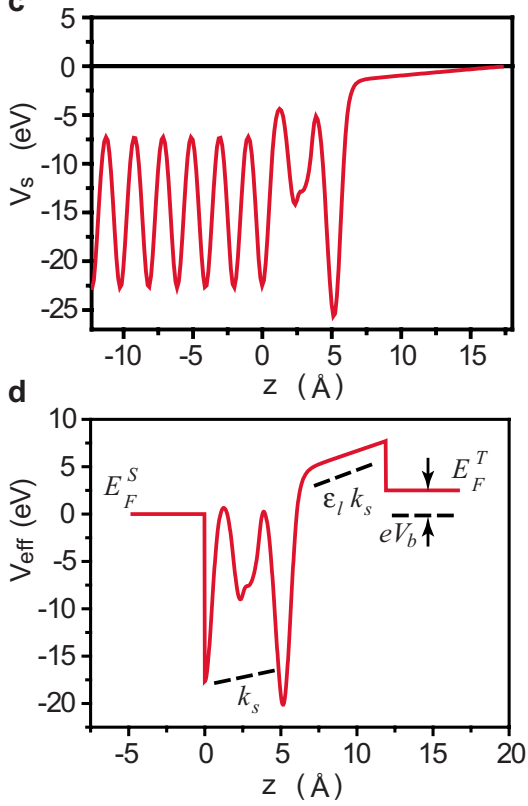

FIG. 5. (Color online) (a) Top view of the $\mathrm{Al}_{2} \mathrm{O}_{3} / \mathrm{NiAl}(110)$ interface structure. (b) Side view of the structure. (c) The calculated $V_{S}(z)$. (d) The calculated $V_{\text {eff }}(z)$.

an oxide dielectric layer chemisorbed on $\mathrm{NiAl}(110)$. The $\mathrm{Al}_{2} \mathrm{O}_{3}$ film on $\mathrm{NiAl}(110)$ has a thickness of about $0.5 \mathrm{~nm}$ with a structure similar to $\gamma-\mathrm{Al}_{2} \mathrm{O}_{3} \cdot{ }^{37}$ The one-dimensional potential across the whole junction is calculated using the structure in Ref. 37 . The NiAl substrate is modeled by a seven-layer slab. The calculations were performed using the VASP code with a cutoff energy of $250 \mathrm{eV}$. The PW91 gradient corrected functional ${ }^{38}$ was used to treat the exchangecorrelation effects. Figure 5 depicts the structure of the $\mathrm{Al}_{2} \mathrm{O}_{3} / \mathrm{NiAl}(110)$ interface together with the calculated onedimensional potential $V_{S}(z)$ and effective potential $V_{\text {eff }}(z)$. A current of $1.0 \mathrm{nA}$ with a bias voltage of $2.5 \mathrm{~V}$ was used in the calculations. As shown in Table II, the separation between the tip and the $\mathrm{NiAl}(110)$ substrate is increased while the vacuum gap distance is reduced when an $\mathrm{Al}_{2} \mathrm{O}_{3}$ layer is included. This can be explained using the same argument above as for the W-tip/ $\mathrm{C}_{60} / \mathrm{Au}(111)$ system. However, the 
TABLE II. The calculated results of the separation distances between the tip and the sample of the W-tip $/ \mathrm{Al}_{2} \mathrm{O}_{3} / \mathrm{NiAl}(110)$ system at $2.5 \mathrm{~V}$ and $1 \mathrm{nA}$. The last two columns list the peak positions of the STM induced light emission spectra.

\begin{tabular}{lccccc}
\hline \hline & $\begin{array}{c}d_{l} \\
(\AA)\end{array}$ & $\begin{array}{c}d \\
(\AA)\end{array}$ & $\begin{array}{c}d_{l}+d \\
(\AA)\end{array}$ & $\begin{array}{c}\lambda_{\text {lowest }} \text { a } \\
(\mathrm{nm})\end{array}$ & $\begin{array}{c}\lambda_{\text {sec low }}{ }^{\mathrm{a}} \\
(\mathrm{nm})\end{array}$ \\
\hline $\mathrm{NiAl}(110)$ & 0.000 & 7.604 & 7.604 & 832 & 634 \\
$\mathrm{Al}_{2} \mathrm{O}_{3} / \mathrm{NiAl}(110)$ & 5.050 & 6.838 & 11.888 & 822 & 634 \\
\hline \hline
\end{tabular}

${ }^{\mathrm{a}} \lambda_{\text {lowest }}$ and $\lambda_{\text {sec low }}$ are the peak positions of the lowest and second-lowest TIP modes in energy, respectively.

shortening of the vacuum gap is larger in comparison with that of the $\mathrm{W}$-tip $/ \mathrm{C}_{60} / \mathrm{Au}(111)$ system because $\mathrm{Al}_{2} \mathrm{O}_{3}$ is an insulator with much lower conductivity relative to $\mathrm{C}_{60}$.

For the calculation of the local-field enhancement factor $G$, the curvature radius of the tip is again chosen to be 3.0 $\mathrm{nm}$ with $b=3.0 \mathrm{~nm}$ and $\phi=45^{\circ}$. The dielectric constant of $\gamma-\mathrm{Al}_{2} \mathrm{O}_{3}$ is chosen to be 3.0 (Ref. 39) while the dielectric function of NiAl is taken from Ref. 40. Figure 6 shows the calculated light emission spectra of the W-tip $/ \mathrm{Al}_{2} \mathrm{O}_{3} / \mathrm{NiAl}(110)$ system with three dominant features. The main feature is a double-peak spectral profile for both cases, relatively sharper for the pristine $\mathrm{NiAl}(110)$ with one peak at $\sim 832 \mathrm{~nm}$ and the other at $\sim 634 \mathrm{~nm}$. These two peaks are considered to be the lowest and the second-lowest modes of TIPs, respectively. ${ }^{41}$ It may be worthy to mention that the height of emission peaks for $\mathrm{NiAl}(110)$ is about onefourth of that for $\mathrm{Au}(111)$, implying a considerably weaker local surface plasmon field for $\mathrm{NiAl}(110)$. The second feature is the pronounced suppression of emission intensity upon the insertion of an $\mathrm{Al}_{2} \mathrm{O}_{3}$ layer because of the weakened local-field enhancement, consistent with the experiment qualitatively. ${ }^{11}$ This is also reflected in the calculated quantum efficiency of about $1.6 \times 10^{-4}$ when a $\mathrm{Al}_{2} \mathrm{O}_{3}$ layer is included, which is about $66 \%$ of the pristine $\mathrm{NiAl}(110)$ substrate. The third feature is the similar spectral profile from the layer-free situation to the $\mathrm{Al}_{2} \mathrm{O}_{3} / \mathrm{NiAl}(110)$ system with a thin film dielectric layer. The peak position for the secondlowest TIP mode remains the same, while a small blueshift of about $10 \mathrm{~nm}$ is observed for the lowest broad TIP mode.

\section{CONCLUSIONS}

In summary, we have investigated the influence of a dielectric layer on STM induced light emission by proposing a combined approach of classical electrodynamics for the field

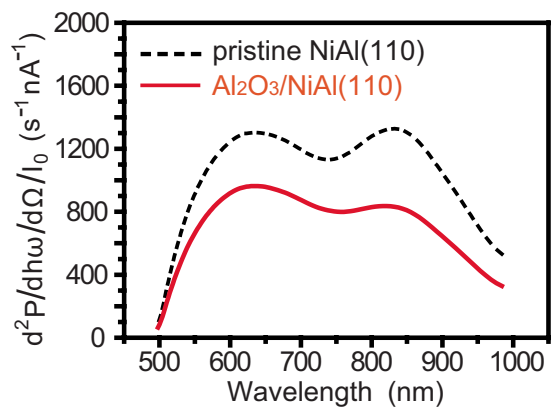

FIG. 6. (Color online) Light emission spectra for the pristine $\mathrm{NiAl}(110)$ (dashed) and the surface covered with a $\mathrm{Al}_{2} \mathrm{O}_{3}$ dielectric layer (solid). The tunneling conditions are $2.5 \mathrm{~V}$ and $1 \mathrm{nA}$. enhancements at the cavity and first-principles calculations to describe the tunneling current-gap distance relation in the system. We treat the response of the junction to the insertion of a dielectric layer from two major aspects: One is the modification of junction geometry (particularly the tip-metal distance), and the other is the modified external field, both in comparison with the layer-free situation.

The calculated radiated powers for W-tip/ $\mathrm{C}_{60} / \mathrm{Au}(111)$ and W-tip $/ \mathrm{Al}_{2} \mathrm{O}_{3} / \mathrm{NiAl}(110)$ indicate that the light emission intensity in both systems is reduced considerably, with the absence of substantial peak shifts with respect to the layerfree situation, in good agreement with most experimental observations. The suppression of the radiated power is mainly due to the increase in the tip-metal separation and resultantly the reduction in the electromagnetic coupling between the tip and the metal substrate. The absence of substantial peak shifts is a compensation result of the blueshift because of the increased tip-metal separation by the redshift of screening of the dielectric layer in the STM cavity.

It is noteworthy that the agreement of our calculated results with experiments is owing to the exact description of the surface potential by the DFT when a dielectric layer is inserted, so that the distance-current dependency can be properly evaluated. The vacuum gap distance between the tip and the layer surface is found to be slightly shortened in spite of the overall increase in the tip-metal separation. Nevertheless, the shortening reported in the present calculation may be underestimated because the density of states of the layer is not taken into account explicitly. Further work can be developed to model STM induced molecular fluorescence, where the electronic structures of both the dielectric layer and the emitting molecule will be considered in the calculation of the transition matrix element.

\section{ACKNOWLEDGMENTS}

We are grateful to Professor G. Kresse for providing the atomic coordinates of the $\mathrm{Al}_{2} \mathrm{O}_{3} / \mathrm{NiAl}(110)$ system. We also thank Y. Zhang and Z. P. Hu for helpful discussions. This work was partly supported by the National Basic Research Program of China (Grant No. 2006CB922003), CAS (Grant No. KJCX2.YW.H06), and NSFC (Grant No. 10574117). 
${ }^{1}$ R. Berndt, J. K. Gimzewski, and P. Johansson, Phys. Rev. Lett. 67, 3796 (1991).

${ }^{2}$ R. W. Rendell and D. J. Scalapino, Phys. Rev. B 24, 3276 (1981).

${ }^{3}$ P. Johansson, R. Monreal, and P. Apell, Phys. Rev. B 42, 9210 (1990).

${ }^{4}$ J. Aizpurua, G. Hoffmann, S. P. Apell, and R. Berndt, Phys. Rev. Lett. 89, 156803 (2002).

${ }^{5}$ R. Berndt, J. K. Gimzewski, and P. Johansson, Phys. Rev. Lett. 71, 3493 (1993).

${ }^{6}$ P. Johansson and R. Monreal, Z. Phys. B: Condens. Matter 84, 269 (1991).

${ }^{7}$ J. Aizpurua, S. P. Apell, and R. Berndt, Phys. Rev. B 62, 2065 (2000).

${ }^{8}$ F. J. Garcia de Abajo and J. Aizpurua, Phys. Rev. B 56, 15873 (1997).

${ }^{9}$ K. Amemiya, Phys. Rev. B 67, 075409 (2003).

${ }^{10}$ R. Berndt, R. Gaisch, J. K. Gimzewski, B. Reihl, R. R. Schlittler, W. D. Schneider, and M. Tschudy, Science 262, 1425 (1993).

${ }^{11}$ X. H. Qiu, G. V. Nazin, and W. Ho, Science 299, 542 (2003).

${ }^{12}$ P. Anger, P. Bharadwaj, and L. Novotny, Phys. Rev. Lett. 96, 113002 (2006).

${ }^{13}$ J. R. Lakowicz, Anal. Biochem. 337, 171 (2005).

${ }^{14}$ T. H. Taminiau, F. D. Stefani, F. B. Segerink, and N. F. Van Hulst, Nature Photon. 2, 234 (2008).

${ }^{15}$ T.-H. Lee, J. I. Gonzalez, J. Zheng, and R. M. Dickson, Acc. Chem. Res. 38, 534 (2005).

${ }^{16}$ J. A. Misewich, R. Martel, Ph. Avouris, J. C. Tsang, S. Heinze, and J. Tersoff, Science 300, 783 (2003).

${ }^{17}$ S. Nie and S. R. Emory, Science 275, 1102 (1997).

${ }^{18}$ K. Kneipp, Y. Wang, H. Kneipp, L. T. Perelman, I. Itzkan, R. R. Dasari, and M. S. Feld, Phys. Rev. Lett. 78, 1667 (1997).

${ }^{19}$ H. Xu, E. J. Bjerneld, M. Kall, and L. Borjesson, Phys. Rev. Lett. 83 , 4357 (1999)

${ }^{20}$ E. Cavar, M.-C. Blum, M. Pivetta, F. Patthey, M. Chergui, and W.-D. Schneider, Phys. Rev. Lett. 95, 196102 (2005).

${ }^{21}$ Z. C. Dong, X. L. Guo, A. S. Trifonov, P. S. Dorozhkin, K. Miki, K. Kimura, S. Yokoyama, and S. Mashiko, Phys. Rev. Lett. 92, 086801 (2004).

${ }^{22}$ J. R. Kirtley, T. N. Theis, J. C. Tsang, and D. J. DiMaria, Phys. Rev. B 27, 4601 (1983).

${ }^{23}$ G. Kresse and J. Furthmüller, Comput. Mater. Sci. 6, 15 (1996).
${ }^{24}$ N. P. Guisinger, N. L. Yoder, and M. C. Hersam, Proc. Natl. Acad. Sci. U.S.A. 102, 8838 (2005).

${ }^{25}$ P. Johansson and G. Wendin, Phys. Rev. B 46, 1451 (1992).

${ }^{26}$ P. Johansson, Phys. Rev. B 46, 12865 (1992).

${ }^{27}$ O. S. Heavens, Optical Properties of Thin Solid Films (Dover, New York, 1965).

${ }^{28}$ L. L. Wang and H. P. Cheng, Phys. Rev. B 69, 165417 (2004).

${ }^{29}$ J. P. Perdew, K. Burke, and M. Ernzerhof, Phys. Rev. Lett. 77, 3865 (1996); J. P. Perdew, K. Burke, and M. Ernzerhof, Phys. Rev. Lett. 77, 3865 (1996); J. P. Perdew, K. Burke, and M. Ernzerhof, Phys. Rev. Lett. 78, 1396(E) (1997).

${ }^{30}$ P. B. Johnson and R. W. Christy, Phys. Rev. B 6, 4370 (1972).

${ }^{31}$ E. P. Palik, Handbook of Optical Constants of Solids (Academic, Orlando, 1985).

${ }^{32}$ W. Y. Ching, M.-Z. Huang, Y.-N. Xu, W. G. Harter, and F. T. Chan, Phys. Rev. Lett. 67, 2045 (1991).

${ }^{33}$ G. Hoffmann, L. Libioulle, and R. Berndt, Phys. Rev. B 65, 212107 (2002).

${ }^{34}$ D. L. Mills, Phys. Rev. B 65, 125419 (2002).

${ }^{35}$ Y. Zhang, X. Tao, H. Y. Gao, Z. C. Dong, J. G. Hou, and T. Okamoto, Phys. Rev. B 79, 075406 (2009).

${ }^{36}$ J. B. Lassiter, J. Aizpurua, L. I. Hernandez, D. W. Brandl, I. Romero, S. Lal, J. H. Hafner, P. Nordlander, and N. J. Halas, Nano Lett. 8, 1212 (2008).

${ }^{37}$ M. Schmid, M. Shishkin, G. Kresse, E. Napetschnig, P. Varga, M. Kulawik, N. Nilius, H. P. Pust, and H.-J. Freund, Phys. Rev. Lett. 97, 046101 (2006).

${ }^{38}$ J. P. Perdew, J. A. Chevary, S. H. Vosko, K. A. Jackson, M. R. Pederson, D. J. Singh, and C. Fiolhais, Phys. Rev. B 46, 6671 (1992).

${ }^{39}$ R. Ahuja, J. M. Osorio-Guillen, J. Souza de Almeida, B. Holm, W. Y. Ching, and B. Johansson, J. Phys.: Condens. Matter 16, 2891 (2004).

${ }^{40}$ J. J. Rechtien, C. R. Kannewurf, and J. O. Brittain, J. Appl. Phys. 38, 3045 (1967)

${ }^{41}$ N. Nilius, N. Ernst, H.-J. Freund, and P. Johansson, Phys. Rev. B 61, 12682 (2000). At high bias voltages such as $5 \mathrm{~V}$, the calculated emission spectra for $\mathrm{NiAl}(110)$ show that the higher-energy band is blueshifted to $496 \mathrm{~nm}$, mainly due to the contribution of higher-energy plasmon modes. 